\title{
Rat embryonic fibroblasts immortalized by MRPS18-2 protein are target for NK-cells
}

\author{
Muhammad Mushtaq ${ }^{1}$, Pradeepa N. Pangigadde ${ }^{1,2}$, Suhas Darekar ${ }^{1}$, Erik Dissen ${ }^{3}$ \\ and Elena Kashuba ${ }^{1,4}$ \\ ${ }^{1}$ Department of Microbiology, Tumor and Cell Biology, Karolinska Institutet, Stockholm, Sweden \\ 2 University "Magna Græcia" of Catanzaro, Viale Europa, Catanzaro, Italy \\ ${ }^{3}$ Department of Anatomy, Institute of Basic Medical Sciences, University of Oslo, Oslo, Norway \\ ${ }^{4}$ R.E. Kavetsky Institute of Experimental Pathology, Oncology and Radiobiology, NASU, Ukraine \\ Correspondence to: Elena Kashuba, email: Elena.Kashuba@ki.se \\ Keywords: mitochondrial ribosomal protein MRPS18-2, cell immortalization, stem cells, NK killing, cytokine expression profile \\ Received: March 16, $2017 \quad$ Accepted: April 24, $2017 \quad$ Published: May 04, 2017
}

Copyright: Mushtaq et al. This is an open-access article distributed under the terms of the Creative Commons Attribution License 3.0 (CC BY 3.0), which permits unrestricted use, distribution, and reproduction in any medium, provided the original author and source are credited.

\section{ABSTRACT}

Overexpression of the human mitochondrial ribosomal protein MRPS18-2 (S18-2) led to immortalization of primary rat embryonic fibroblasts (REFs). The derived cells (18IM) expressed embryonic stem cell markers. Noteworthy, genes encoding the coX family proteins were up-regulated significantly. It is known that the coX family proteins are involved in the regulation of immune response.

In the present work we demonstrate that 18IM cells behave like stem cells when subjected to directed differentiation in vitro. However, unlike stem cells, 18IM cells do not develop tumors in vivo, in SCID mice. This phenomenon is observed due to the strong natural killer (NK) cell immunogenicity. 18IM cells were better recognized by NK cells, compared with primary REFs, as was shown by a standard NK killing assay. Our data explain asymmetry in behavior of stem-like cells in vivo and in vitro, and this support the notion that stem and/or cancer-initiating cells are preferred targets for NK-cells. Concluding, the S18-2 protein is a putative target for cancer vaccines.

\section{INTRODUCTION}

The S18 family of mitochondrial ribosomal proteins (MRPS18, S18 in the text) consists of three proteins, S18-1-3 [1]. S18 proteins show low sequence homology to each other (about 20 percent), suggesting various functions, as uncharacterized yet.

We have found that overexpression of the S182 protein (NP_054765) resulted in immortalization of primary rat embryonic fibroblasts (REFs), associated with induction of stem cell marker expression [2]. In the established cell line (18IM) genes, encoding proteins of the cyclooxygenase (COX) and NADH ubiquinone oxidoreductase (NDUF) families, were significantly up-regulated. It is known that COX and NDUF family proteins are involved in the control on cell proliferation, oxidative phosphorylation, cellular respiration, and other redox reactions. This indicates that $18 \mathrm{IM}$ cells are more active metabolically than REFs. The cellular pathways characteristic for rapidly proliferating cells were also activated in 18IM cells, namely the ubiquinone (Coenzyme Q10) biosynthesis, fatty acid elongation in mitochondria, and PI3K/AKT signaling [3]. When grown at a high confluence, 18IM cells demonstrated characteristics of stem cells by differentiating into fat cells spontaneously. Moreover, a proportion of the cells started to express the beta-III-tubulin, MHC class II, and pan-keratin [2].

The 18IM line was not tumorigenic upon inoculation into experimental animals, severe combined immunodeficient (SCID) mice, despite the demonstrated phenotype of the fast proliferating immortalized cells. Actually, small tumors were detected in the first 7-14 days. However, tumors regressed rather fast, in a few weeks in all of animals [3], [4]. Therefore, we asked a question, what are the factors inhibiting growth of 18IM cells in vivo? Could the S18-2 protein expressed at the elevated levels evoke immune response? 
In the present paper we aimed to explain asymmetry in 18IM growth in vivo and in vitro.

\section{RESULTS}

\section{Directed differentiation of $18 \mathrm{IM}$ cells}

As was mentioned above, 18IM cells resembled the stem cells and spontaneously differentiated under confluent growth conditions [2]. We aimed to determine whether 18IM cells could be subjected to so-called "directed" differentiation. Accordingly, to promote neurogenic differentiation, 18IM cells were treated with retinoic acid (RA). The first phenotypic changes were observed as early as $48 \mathrm{~h}$ after the treatment (Figure 1A). After 1 week, 18IM showed the "glial cell-like" morphology with characteristic filopodia. It is noteworthy that cells proliferated to a certain confluence for approximately 3 weeks, after which a proportion of cells died. A month later, most of the 18IM cells cultured with RA died, as expected for differentiated primary cells. To confirm differentiation into glial cells, after treatment with RA for one week 18IM cells were stained with antibodies against MAP-2 and nestin (Figure 1B). Cells showed strong MAP-2 and nestin signals, suggesting that 18IM cells differentiated into precursors of neuronal cells. These markers showed very weak signal on untreated 18IM cells.
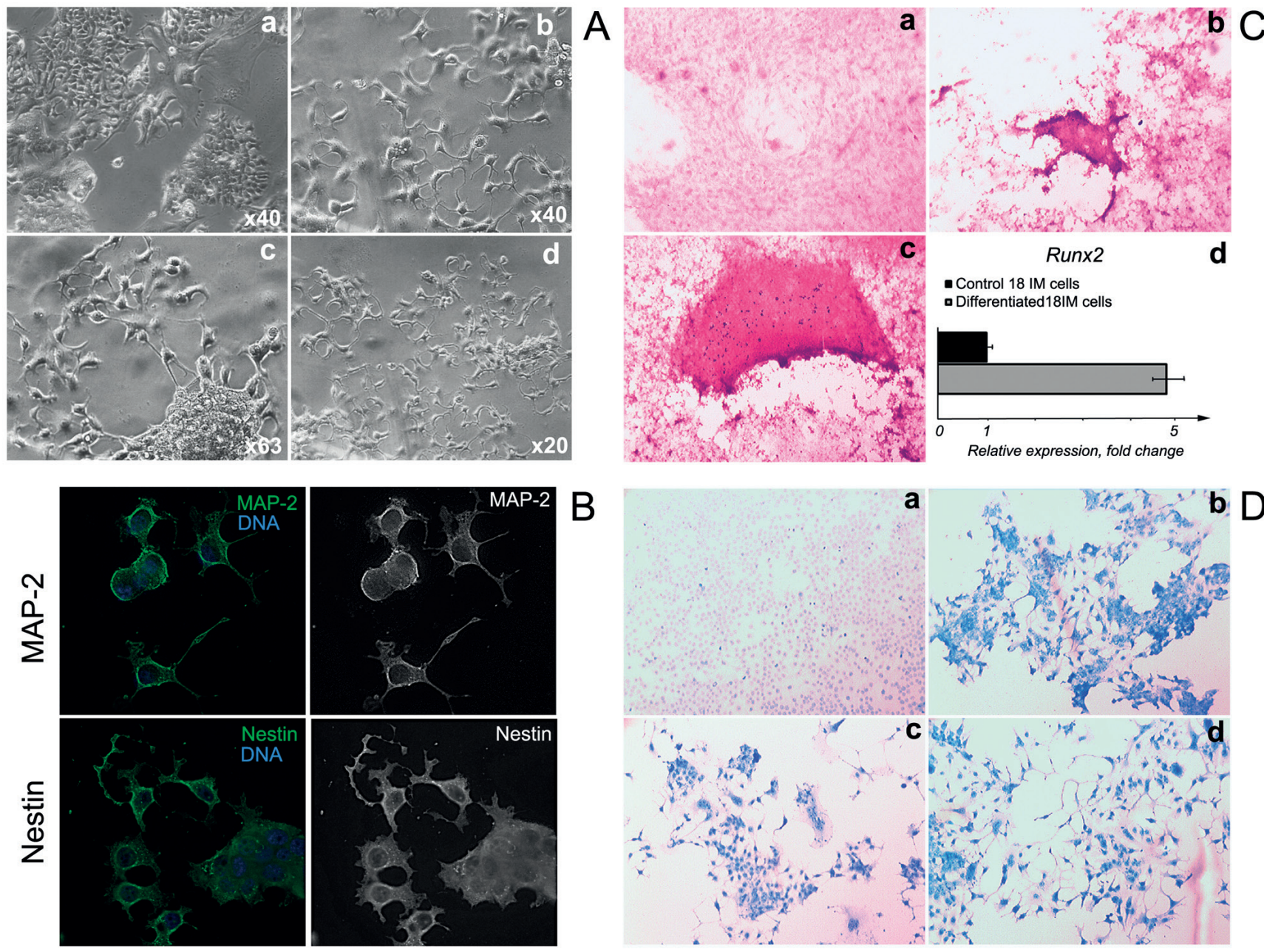

Figure 1: Directed differentiation of 18IM cells in vitro. A. - 18IM cells grown on coverslips were treated for 1 week with 20 $\mu \mathrm{M}$ of RA in culture medium; a - control 18IM cells; b-d, differentiated cells. Notice the change of morphology after treatment with RA. B. - 18IM cells were differentiated as in (A). Upper panel - immunostaining with anti-MAP-2 antibody (green); bottom panel - with antinestin antibody (green). DNA is shown in blue. Objective was x63. C. - 18IM cells were differentiated with a cocktail of dexamethasone, ascorbic acid-2-phosphate, and glycerol-2-phosphate in culture medium for 4 weeks: a - untreated 18IM cells; b-c - differentiated cells, both stained with Alizarin Red S. The strong red signal suggests mineralization of the osteogenic cells. Objective was $\mathrm{x} 40 ; \mathrm{d}-$ Runx $2 \mathrm{expression}$ at the mRNA level, assessed by q-PCR. D. - 18IM cells were differentiated for 4 weeks upon treatment with $0.5 \mu \mathrm{M}$ and $1 \mu \mathrm{M}$ of the H-89 in culture medium. The untreated 18IM cells are shown in panel a, and differentiated cells are shown in panels b-d; both are stained with Alcian Blue. Bright blue staining and a change in cell morphology are seen in differentiated cells (panels b-d). Objective was x63. 
To evoke osteogenic differentiation, cells were cultured in the medium, which contained ascorbic acid2-phosphate, glycerol, and dexamethasone. Cells grown on ordinary Iscove's modified Dulbecco's medium (IMDM) were used as internal controls. To monitor mineralization, cells were stained with a solution of Alizarin Red S. Strong signal was detected on a proportion of $18 \mathrm{IM}$ cells after differentiation (Figure 1C, panels $b$ and c), by contrast with the control 18IM cells (Figure $1 \mathrm{C}$, panel a). The dark red signal was somewhat diffuse because of the use of the Alizarin Red solution at the low $\mathrm{pH}$ (4.6). Under such conditions, the partial removal of calcification in tissues has been observed [5]. To confirm osteogenic differentiation, expression levels of the Runx2 (NM_001278483) were assessed by Q-PCR. Runx2 encodes a transcription factor that is essential for the maturation of osteoblasts and is expressed at higher levels upon osteogenic differentiation [6]. Runx2 expression was elevated significantly in differentiated $18 \mathrm{IM}$ cells (Figure $1 \mathrm{C}$, panel d).

To induce chondrogenesis, 18IM cells were grown in a medium supplemented with $\mathrm{H}-89$. We found that cell morphology was changed significantly after 4 weeks (Figure 1D, b-d). To monitor negatively charged molecules, such as RNA, DNA, glycosaminoglycans, and proteoglycans, cells were stained with Alcian Blue solution. As seen on Figure 1D, 18IM cells were negative for Alcian Blue staining (panel a), while cells showed an intense blue signal after H-89 treatment (Figure 1, panels b-d). Blue dye was detected in the cytoplasm and membranes of the differentiated cells, suggesting the production of acid glycosaminoglycans that are the elements of the extracellular matrix.

Hence, the 18IM cells demonstrated features of stem cells. We wondered whether 18IM cells differs from the primary REFs also by expression profiles of inflammatory cytokines and receptors, that is characteristic for the stem and cancer-initiating cells.

\section{Expression pattern of inflammatory cytokines and receptors}

To analyze the expression of genes related to the different inflammatory pathways at the mRNA level, the $\mathrm{RT}^{2}$ profiler assay was performed, using RNA isolated from 18IM cells and REFs for comparison. After an analysis of the quantitative PCR (q-PCR) data, genes that showed at least 4 folds difference in expression levels between 18IM and REFs were selected. Genes on the plate represented mainly chemokines, cytokines and their receptors. 26 genes out of 84 analyzed were expressed in $18 \mathrm{IM}$ at the higher levels compared with REF (Table 1, Figure 2A, and Supplementary Table 1). All of these genes encode proteins that induce pro-inflammatory effects.

It is well known that the major histocompatibility complex (MHC) class I molecule, one of the most important players involved in immune response, is expressed at lower levels in stem and cancerous cells [7]. MHC class I molecule expression levels were measured, using a specific anti-MHC-I antibody. Indeed, levels of MHC class I protein on 18IM cells were significantly
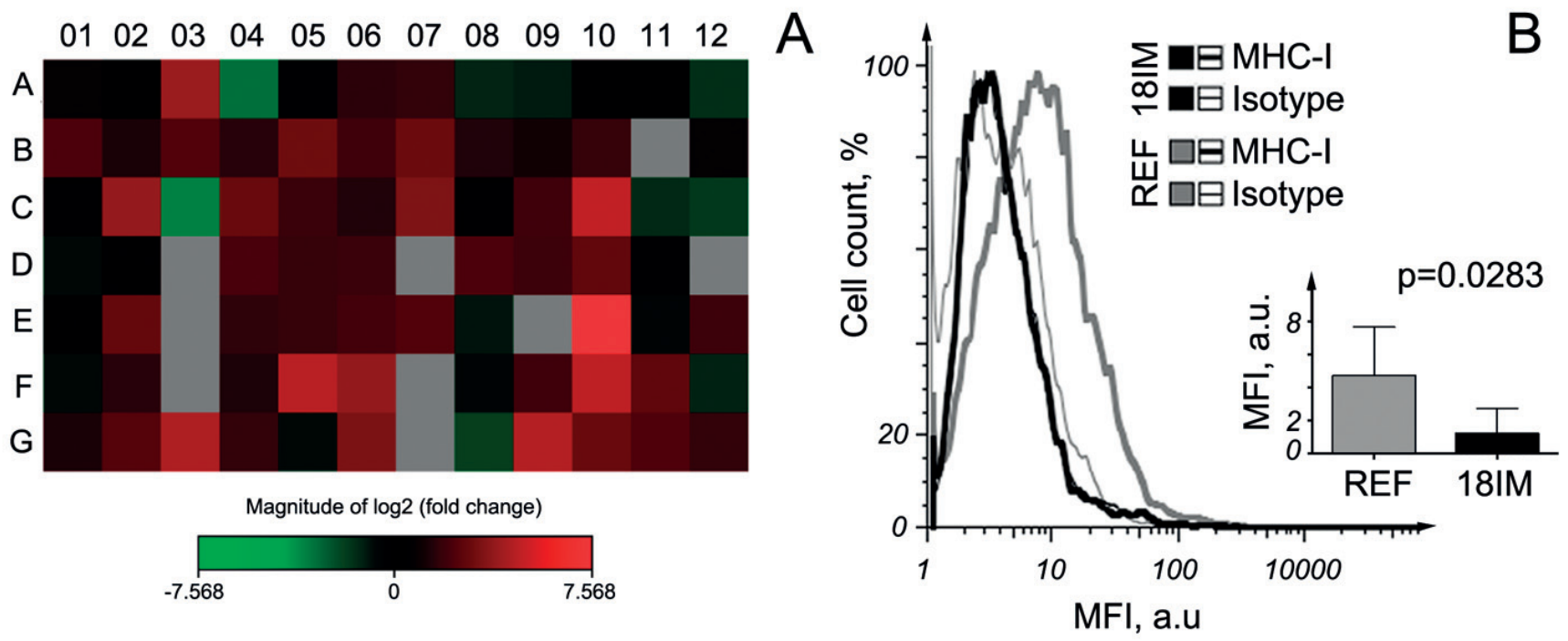

Figure 2: Expression of chemokines and their receptors in 18IM cells, compared with REFs. A. - The heat map of the fold changes in gene expression between 18IM cells and REFs for every gene in the array, in the context of the array layout (see Figure S1 for the genes). B. - 18IM cells and primary REFs were incubated with either anti-MHC-I or unrelated isotype control antibodies. A representative FACS analysis showing the difference in MHC-I molecule expression between REFs and 18IM cells; the inserted panel shows statistical analysis of MHC-I expression from three independent experiments, using two tailed paired T test on the combined mean values. MFI - the mean fluorescence intensity; a. u. - arbitrary units. 
Table 1: The list of cytokines, chemokines and their receptors that were expressed at the mRNA levels at $\geq 4$ fold in 18 IM cells, compared with REFs.

\begin{tabular}{|c|c|c|c|}
\hline \multicolumn{4}{|c|}{ Genes upregulated in 18IM cells compared with REFs } \\
\hline Cytokine/Receptor & Accession number & Binding receptor/cytokine & Relative fold change \\
\hline $\mathrm{Ccll1}$ & NM_019205 & CCR2, CCR3, CCR5 & 15.73 \\
\hline $\mathrm{Ccl5}$ & NM_031116 & CCR1, CCR3, CCR5 & 4.58 \\
\hline $\mathrm{Ccl} 7$ & NM_001007612 & CCR1, CCR2, CCR3 & 4.94 \\
\hline Ccrl & NM_020542 & CCL3, CCL5, CCL7, CCL23 & 7.92 \\
\hline Ccr2 & NM_021866 & CCL1 & 7.59 \\
\hline Csfl & XM_008761426 & CSF1R (CD115) & 14.57 \\
\hline Csf3 & NM_017104 & CSF3R & 7.20 \\
\hline Cxcl1 & NM_030845 & CXCR2 (IL8RB, IL8R2) & 9.99 \\
\hline Cxcl12 & NM_022177 & CXCR4, CXCR7 & 28.78 \\
\hline Cxcr5 & NM_053303 & CXCL13 & 4.47 \\
\hline Il11 & NM_133519 & IL11RA & 4.49 \\
\hline Il15 & NM_013129 & IL15A & 6.31 \\
\hline$I l 17 f$ & NM_001015011 & IL17RA? & 6.61 \\
\hline Il1rn & NM_022194 & IL1R1 & 4.10 \\
\hline Il21 & NM_001108943 & IL21R & 4.91 \\
\hline Il2rg & NM_080889 & $\begin{array}{l}\text { IL2RA，IL4RA，IL7RA，IL9RA, } \\
\text { IL15RA, IL21RA }\end{array}$ & 189.76 \\
\hline Il6st & NM_001008725 & IL6:IL6RA, IL6/IL12 complexes & 25.97 \\
\hline Il7 & NM 013110 & IL7R, TCRß, HGF & 13.92 \\
\hline $\operatorname{Tn} f_{S f 10}(T R A I L)$ & NM_145681 & $\begin{array}{l}\text { TRAILR1 (DR4), TRAILR2 (DR5), } \\
\text { TRAILR3, TRAILR4 }\end{array}$ & 9.72 \\
\hline $\operatorname{Tn} f s f 13 b$ & NM_001109112 & $\begin{array}{l}\text { TNFRSF13B (TACI), TNFRSF17 } \\
\text { (BCMA), TNFRSF13C (BAFF-R) }\end{array}$ & 23.46 \\
\hline $\operatorname{Tn} f_{S f 14}$ & NM_001191803 & TNFRSF14 & 7.29 \\
\hline Gene name & Accession number & Description & Relative fold change \\
\hline Mif & NM_031051 & MIF, MHC class II & 27.82 \\
\hline Spp1 & NM_012881 & $\begin{array}{l}\text { This gene encodes osteopontin, or } \\
\text { secreted phosphoprotein }\end{array}$ & 21.81 \\
\hline $\operatorname{Tn} f_{s} f 4$ & NM_053552 & $\begin{array}{l}\text { This gene encodes glycoprotein } \\
\text { Gp34 }\end{array}$ & 4.77 \\
\hline \multicolumn{4}{|c|}{ Genes downregulated in REFs compared with $18 I M$} \\
\hline Cytokine/Receptor & Accession number & Binding receptor/cytokine & Relative fold change \\
\hline Ccl12 & NM_001105822 & CCR2 & 8.64 \\
\hline $\operatorname{Csf2}$ & NM_053852 & CSF2R (CD116) & 11.29 \\
\hline
\end{tabular}

lower than those measured on their REF counterparts (Figure 2B).

The decreased levels of MHC class I molecules on the surface of virus-infected and cancer cells make them targets for NK cells, we wondered whether this also apply for 18IM cells.

\section{IM cells showed higher susceptibility for natural cytotoxicity}

To determine whether 18IM cells show susceptibility to recognition and killing by NK cells, an in vitro NK cell killing assay for 18IM cells in comparison with primary REFs was performed, using rat splenocytes. REFs described earlier [2] and rat splenocytes, used in this study, were both derived from the Sprague Dawley (SD) rats; hence, 18IM cells, REFs, and splenocytes could be considered as isogenic.

Initially, REFs and 18IM cells were presented to the naïve NK cells (i.e., not activated). No significant differences were observed in the killing pattern of REFs and 18IM cells (Figure 3A). By contrast, when splenocytes were activated with interleukin 2 (IL-2), their recognition of 18IM cells and REFs was dissimilar (Figure 3B). 18IM cells showed higher susceptibility to NK-recognition, compared with REFs. A cytotoxic effect was observed at even low splenocyte/rat cell (E: T, Effector: Target) ratios, suggesting that the cytotoxic reaction occurs also in anatomical compartment where NK cells are poorly represented. 
To determine the mechanism responsible for the observed natural cytotoxic effect, a specific antibody against activating receptor NKp46 (or the control antibody, isotype matched) was added to the lymphocytotoxicity assays. As shown in Figure 3C, no change in REF lysis was observed. On contrary, treatment with anti-NKp46 antibody, but not with the isotype control antibody, prevented the selective killing of 18IM target cells (Figure 3D).

Taking into consideration that 18IM killing was mediated by NK cells, we asked a question whether this process may take place in experimental animals, SCID mice.

\section{IM cells were recognized by NK cells of SCID mice}

To find out whether 18IM cells could be recognized and killed by NK cells of experimental animals (SCID mice), in vitro NK cell killing assay was performed, using splenocytes isolated from SCID mice. Three experiments for 18IM cells and REFs were performed, and for one experiment a pool of the activated NK cells from two spleens were used. NK cells of SCID mice killed 18IM successfully, in comparison with REFs (Figure 4A).

The next question was whether NK cells could be detected in proximity to 18IM cells in SCID mice. Therefore, 18IM cells were inoculated into SCID mice subcutaneously and after 1 week the tissue was isolated. Tissue sections were stained with antibodies against S182 and NKp46. The strong signal of NKp46 was detected on the membrane of small cells (Figure 4B, brown) at the inoculation site.

Next, we have diminished expression of the S182 in 18IM cells, using a cocktail of siRNAs for $24 \mathrm{~h}$ (see Supplementary Figure S1 online). We have to mention, that $18 \mathrm{IM}$ cells were treated with the mixture of siRNAs only for $24 \mathrm{~h}$, because the longer treatment $(\geq$ $48 \mathrm{~h}$ ) induced the cell death, as we have shown earlier [2]. The treated cells were introduced into SCID mice
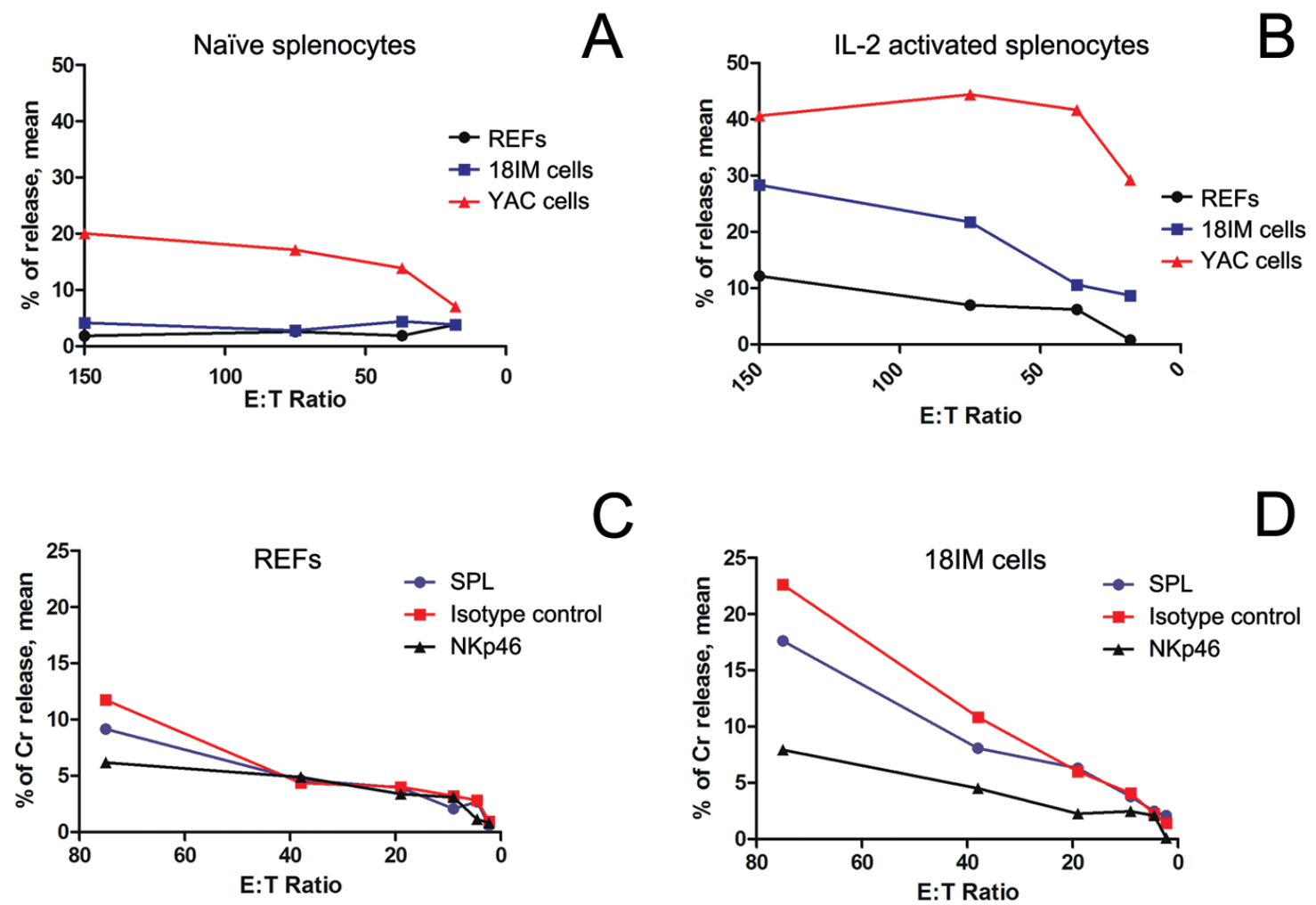

Figure 3: The cytotoxic recognition of REFs and 18IM cells by splenocytes, studied at various splenocyte-to-targetcell $(\mathbf{E}: \mathrm{T})$ ratios. Rat splenocytes were used as effector $(\mathrm{E})$ cells, and REFs and 18IM cells as targets (T) in the assay. Non-parametric t-test (panels A and B) and Wilcoxon signed rank test (C and D) were used to compare a median of three different experiments, performed in triplicates for all the E:T ratios. A. - No differences were observed between REFs and 18IM cells for naïve splenocytes $(p=0.0747)$. YAC - control mouse lymphoma YAC-1 cell line. B. - The IL-2-activated rat splenocytes preferentially recognize 18IM cells, compared with primary REFs $(p=0.0305)$. C. - NKp46 blocking assay for REF recognition by activated splenocytes: SPL treated with anti-NKp46 antibody (NKp46, $p=0.0938)$, and SPL treated with the isotype control antibody (isotype control, $p=0.0625)$. D. - NKp46 blocking assay for $18 \mathrm{IM}$ cell recognition by activated splenocytes: SPL treated with anti-NKp46 antibody (NKp46, $p=0.0320$ ), and SPL treated with the isotype control antibody (isotype control, $p=0.0625$ ). 

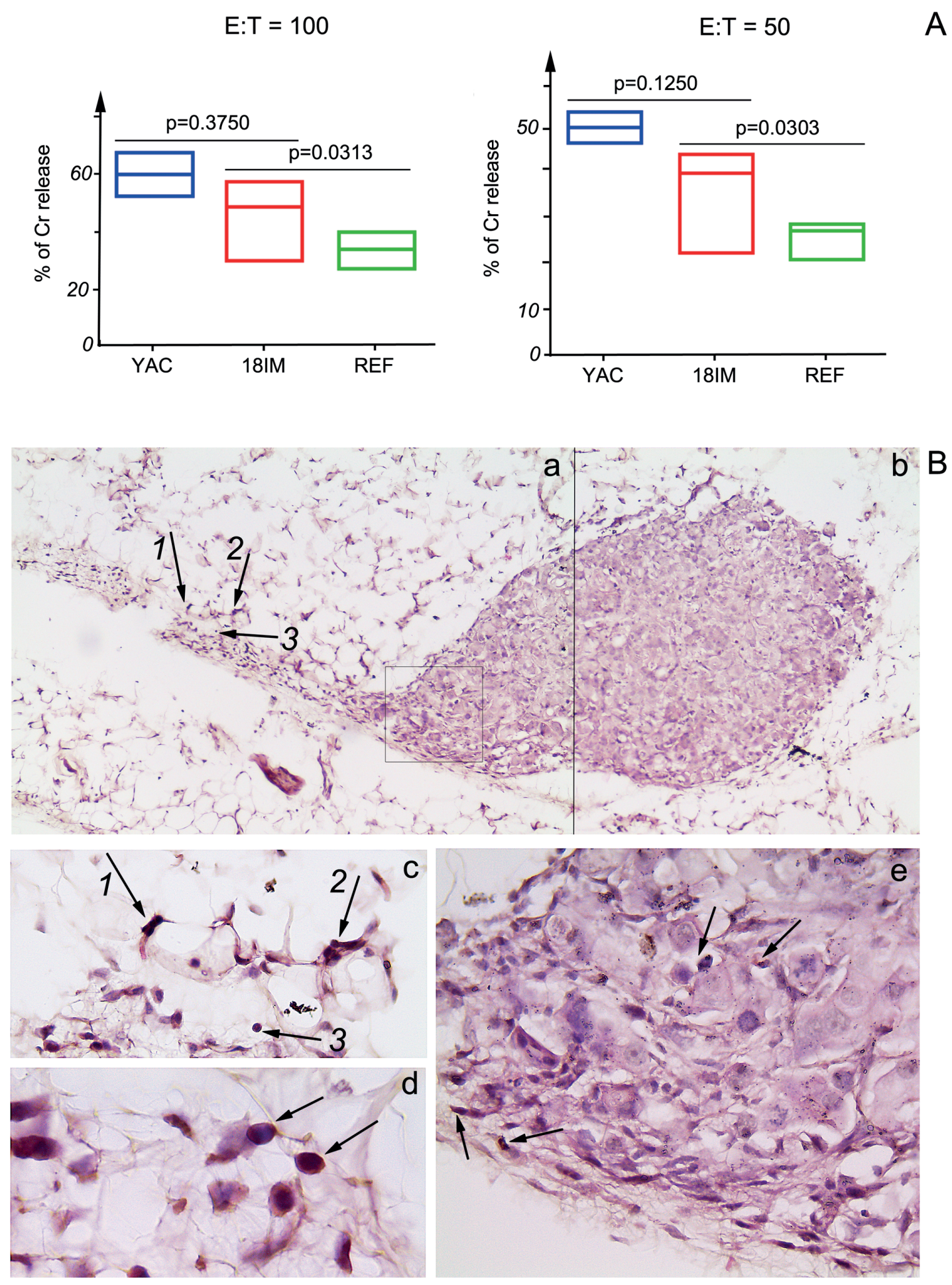

Figure 4: Recognition of 18IM cells by NK-cells of SCID mice in vitro and in vivo. A. - Splenocytes of SCID mice were used as effector (E), and 18IM cells and REFs as targets (T) in the in vitro NK cell cytotoxicity assay. The cytotoxic recognition was studied at E:T ratios 100:1 and 50:1. Non-parametric t-test was used to compare a median of three different experiments, and for one experiment a pool of the activated NK cells from two spleens was used. 18IM cells were killed by the IL-2 activated NK cells more efficiently than REFs $(p=0.0313$ for $\mathrm{E}: \mathrm{T}=100: 1$ and $p=0.0303$ for $\mathrm{E}: \mathrm{T}=50: 1)$, while no significant differences were observed between 18IM and the control mouse lymphoma YAC-1 (YAC) cells killing. B. - Tissue sections show a bulk of 18IM cells and NKp46-positive cells in the proximity of the former (black arrows on the panels a, c, d, and e). Panels a and b are merged from the two sequentially captured images with objective $\mathrm{x} 10$. Black arrows with numbers on the panel a correspond to those on the panel $\mathrm{c}$ (the image on $\mathrm{c}$ was captured with objective $\mathrm{x} 40$ ). Notice the cytoplasmic NKp46 signal (brown) on the panel d (objective was x100). 18IM cells that show S18-2 signal (pink) on the panel e were captured with objective $\mathrm{x} 40$ (the image corresponds to cells framed in the square on the panel a). 
subcutaneously, and the animals were observed for 6 weeks. In contrast to the established 18IM cells, the formation of small tumors was detected in all 4 spots (in two mice), along with inflammation (see Supplementary Figure S2 online, left and right panels, respectively).

\section{DISCUSSION}

NK-cells, a subset of innate lymphoid cells (ILCs), express both, inhibitory and activating receptors on their surface. The inhibitory receptors (KIRs in human, Ly49 in rodents) recognize MHC class I or MHC class I-like molecules. Activating receptor NKG2D (KLRK1, NP_031386) binds to stress-inducible activating ligands, such as MIC-A, MIC-B, and ULBPs in humans and RAE, H60, and MULT1 in mice, respectively [8]. However, the NKp46 ligands (human NCR1, NP_001138929) are still poorly characterized in both species. When the equilibrium between inhibitory and activating receptors is shifted, downregulation of MHC class I expression or the presence of activating ligands induces NK-cells to kill the targets. Malignant transformation and viral infection result in decreased expression of MHC class I molecules, as a rule. Rapid killing of tumor cells that were deficient in MHC class I molecules was observed both in vivo and in vitro [9], [10], [11]. Noteworthy, NK cells preferentially target human cancer-initiating cells in vitro [12], [13].

Stem cells, especially embryonic stem cells (ESC) form teratomas in experimental animals and tumor growth depends on the immune response of the host. It was shown, that NK-cells could inhibit (partially) growth of tumors, formed by mouse ESCs. The tumor regression was observed in one out of 14 animals upon inoculation of $1 \cdot 10^{6}$ of ESCs into SCID mice [14]. Moreover, rat NK cells (isolated from spleen) killed efficiently ESCs in contrast to their differentiated counterparts, as was shown by the chromium release assay in vitro [14]. Noteworthy, the MHC class I was expressed at very low levels on the surface of ESCs, while a reciprocal expression pattern was observed for the ligands for the activating receptor

\section{Non-limited cell proliferation}

\section{In vitro}
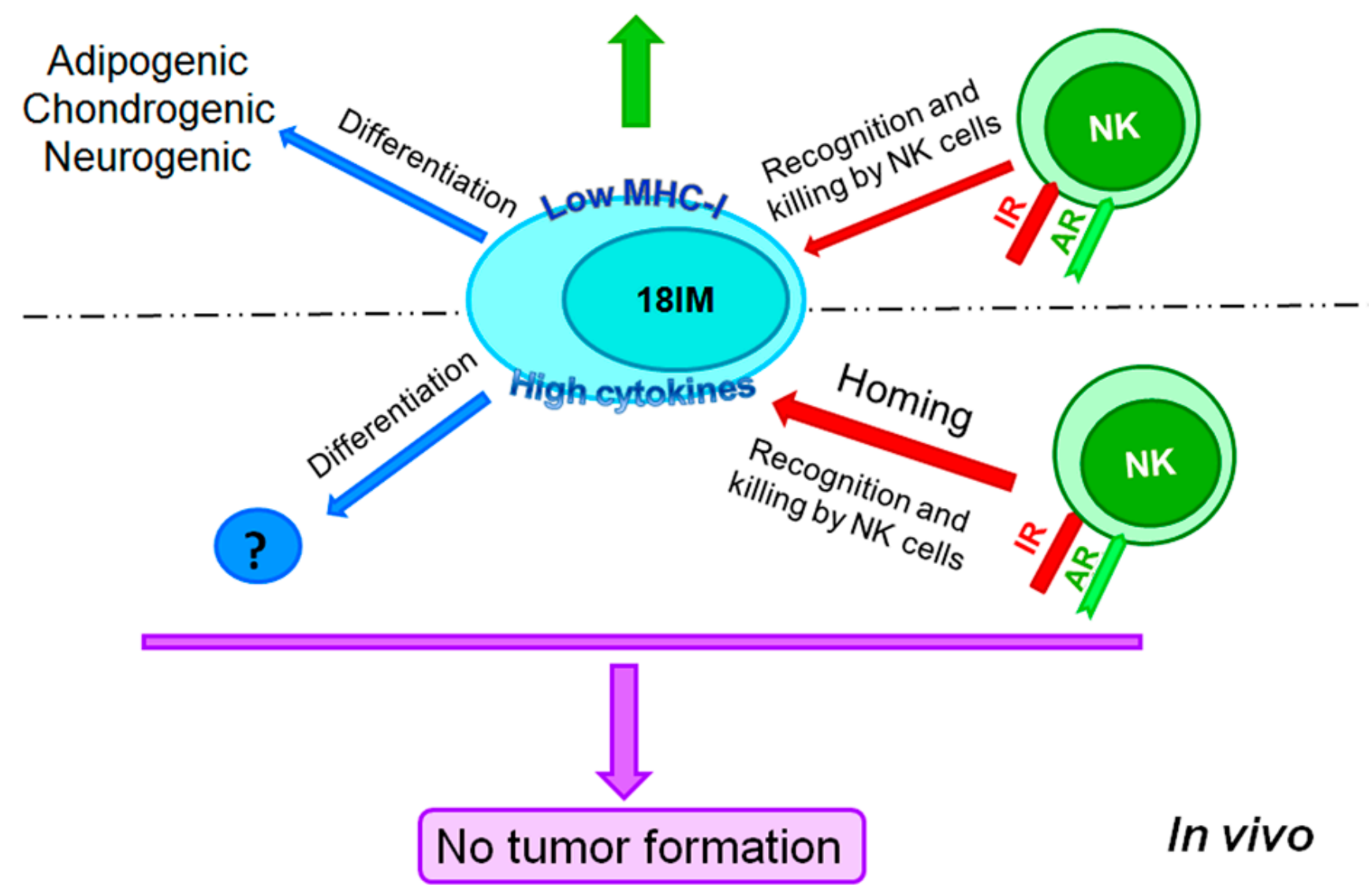

In vivo

Figure 5: 18IM cells are immortalized but do not produce tumors in experimental animals, showing the asymmetric behavior in vitro and in vivo. 18IM cells are recognized (and killed, eventually) by NK cells of SCID mice. They can be transdifferentiated after inoculation as well. AR - activating receptors on NK cells; IR - inhibitory receptors on NK cells. 
NKG2D [7]. Similar phenomenon was observed with human induced pluripotent stem cells (hiPSCs): IL-2 activated NK-cells recognized and killed the former, and this event was partially regulated by the activated NK-cell receptor DNAM-1 (CD226, NP_001290547). Moreover, hiPSCs expressed CD112 (PVRL2, NP 001036189) and CD155 (PVR, NP_001129240) proteins that are ligands for DNAM-1 [15]. Interaction between NK-cell activating receptor NKG2D and its ligands, MICA (NP_000238) and MICB (NP_001276089) played an important role in eradication of hiPSCs.

Our results show that the $18 \mathrm{IM}$ cells, resembling ESCs, were not tumorigenic in experimental animals, SCID mice, due to their NK susceptibility and probable trans-differentiation.

It is known that chemokines regulate various biological functions of $\mathrm{NK}$ cells. Like IL2, CC chemokines can induce the proliferation and activation of killer cells (see ref. [16]). Chemokines activate NK cells to become the highly cytolytic cells, known as the CC chemokine-activated killer (CHAK) cells that are able to kill tumor cells [17]. It was shown that certain chemokines are important for the CHAK activity of NK cells [18]. The CCL5, CCL7, CCL11, CXCL1, CXCL12, CCR1, and CCR2 were listed as activating chemokines or their receptors [19].

Noteworthy, we found that these molecules were also expressed in 18IM at the significantly higher levels, compared to REFs. This may add further evidences that can explain why $18 \mathrm{IM}$ cells were preferentially targeted by NK cells, compared with REFs.

Importantly, NK cells were detected at the inoculation site after 7 days of grafting, as was shown by immunohistochemistry. We may speculate that the chemokine and chemokine receptors expressed at the surface of 18IM cells induced homing of NK cells to the introduced cells. Thus, it was shown before, that NK cells were recruited towards CCL2 and CCL5 [20], and CXCL10, together with CXCL11 directed NK cells to the MHC class I deficient cells [21]. CX3CL1 and CX3CR1 that were also upregulated in 18IM cells were shown to be involved in eradication of metastasis [22]. When the levels of S18-2 protein were diminished (after the treatment with the specific siRNAs), the small tumors were formed. Hence, we may conclude on that non-tumorigenic behavior of 18IM cells in SCID mice is due to activity of NK cells, activated by chemokines and their receptors expressed by 18IM cells.

Beside activation of NK cells, 18IM cells can also be differentiated by treatment with cocktails of various chemicals, not only spontaneously, as we found earlier [2]. Moreover, the decreased expression of surface MHC class $\mathrm{I}$, the main inhibitory molecule for NK cells recognition was shown for the 18IM cells (summarized on Figure 5).

Our data allows us speculate that the S18-2 protein plays an important role in carcinogenesis and modulation of the cell immunogenicity. The molecular mechanisms of cell immortalization upon overexpression of S18-2 and the role of S18-2 in cell cycle control and carcinogenesis are under current investigation.

The susceptibility of S18-2-transfected cells to NK cells suggests that NK cells may be involved in immune intervention in cancers overexpressing S18-2. These new findings will open new avenues of research and have important implications for both basic and clinical science.

\section{MATERIALS AND METHODS}

\section{Cell culture and immunostaining}

Primary REFs and 18IM cells (i.e., REFs, immortalized by the overexpression of S18-2 [2]) were used in the present study. All cells (except for those used in differentiation studies) were cultured at $37^{\circ} \mathrm{C}$ in Iscove's medium that contained $10 \%$ fetal bovine serum and appropriate antibiotics.

To diminish the S18-2 levels, a mixture of the four siRNAs was used (Thermo Scientific), according to the manufacturer's protocol, as described in details earlier [2]. Each of 4 siRNA could target the coding region of $S 18$ 2. The cell lysates were prepared, using NP40-containing buffer (see [2] for details). After western blotting (using $12 \%$ SDS-polyacrylamide gel), membranes were stained with rabbit polyclonal antibodies against anti-S18-2 (Proteintech Group, Inc.) and GFP (Cell Signaling Technology), and also with mouse monoclonal anti-actin antibody (Sigma-Aldrich). To visualize protein bands, the ECL kit, anti-mouse and anti-rabbit horseradish peroxidase conjugated secondary antibodies (produced in sheep and donkey, correspondingly) were used (GE Healthcare BioSciences AB).

Before staining, cells were grown on coverslips in six-well plates and fixed in a mixture of methanol and acetone $(1: 1)$ at $-20^{\circ} \mathrm{C}$. Cells were rehydrated in phosphate-buffered saline (PBS) and stained. The following primary antibodies were used: anti-nestin and anti-MAP-2 (Cell Signaling Technology), and conjugated with fluorochrome PE the OX-18 (Novus Biologicals). FITC-conjugated rabbit anti-mouse serum (Dako) was used as a secondary antibody. Hoechst 33258 (SigmaAldrich) was added at a concentration of $0.4 \mu \mathrm{g} / \mathrm{mL}$ in the secondary antibody solution for DNA staining. Images were captured using a Leitz DM RB DAS microscope fitted with a dual-mode cooled charge-coupled device (CCD) camera (C4880, Hamamatsu).

\section{Directed differentiation}

To evoke the neurogenic differentiation, 18IM cells were treated with RA solutions in DMSO, and 
the final concentration of RA was $10 \mu \mathrm{M}$ and $20 \mu \mathrm{M}$, correspondingly.

To perform osteogenic differentiation, 18IM cells and the control REFs were grown in IMDM medium, supplemented with dexamethasone $(0.1 \mu \mathrm{M})$, ascorbic acid-2-phosphate (0.2 mM), and glycerol-2-phosphate (10 $\mathrm{mM}$ ), as described previously [23]. Growth medium was changed three times per week. After 2 and 4 weeks, cells were stained with a $2 \%(\mathrm{w} / \mathrm{v})$ solution of Alizarin Red S (Sigma-Aldrich), which forms complexes with the calcium ions produced by osteocytes [24]. The $\mathrm{pH}$ of the solution was adjusted to 4.6 , using $10 \%$ ammonium hydroxide. Cells were incubated for $30 \mathrm{~min}$ in the Alizarin Red S solution and washed six times with water. To monitor chondrogenic differentiation, cells were grown in Glasgow minimum essential medium (G-MEM) supplemented with nonessential amino acids, sodium pyruvate, and $0.1 \mathrm{mM}$ beta-mercaptoethanol, according to the method described previously [4]. The N-(2-(4-bromocinnamylamino) ethyl)-5-isoquinolinesulfonamide (H-89), an inhibitor of a protein kinase $\mathrm{A}$, was used previously to induce chondrogenesis of rat mesenchymal stem cells at 0.1-1.0 $\mu \mathrm{M}$ [25]. In our experiments, the medium in two wells contained $0.5 \mu \mathrm{M}$ of $\mathrm{H}-89$ (Adipogen International), and in two other wells, $1 \mu \mathrm{M}$ of H-89. Cells in the remaining two wells were used as negative controls. After 4 weeks, cells were stained with Alcian Blue (Sigma-Aldrich). Cells were incubated in a solution of $1 \%(\mathrm{w} / \mathrm{v})$ Alcian Blue in $3 \%$ acetic acid (the $\mathrm{pH}$ of the staining solution was 2.5 ) for $30 \mathrm{~min}$ as previously described [26]. Cells were then washed six times with water.

\section{Quantitative PCR (q-PCR)}

Total RNA was isolated from cells before and after osteogenic differentiation, using an RNeasy Mini kit (Qiagen Inc). Approximately $1 \mu \mathrm{g}$ of total RNA was used for cDNA synthesis, using a First Strand Synthesis Kit (Sigma-Aldrich), according to the manufacturer's protocol. Primer concentration was adjusted to a final concentration of $3 \mu \mathrm{M}$. Total reaction volume for all q-PCR experiments was $20 \mu \mathrm{l}$. Q-PCR was performed, using an SYBR Green Master Mix on a 7900 machine (Applied Biosystems, Foster City, CA, USA). The following primers for the rat Runx2 were used: Runx2_For 5'-ACACCGTGTCAGCAAAGC-3'; Runx2_Rev 5'-GCTCACGTCGCTCATCTTG-3'. As an internal control for standardizing expression, a gene encoding TATA-Binding protein ( $T b p$, NM_001004198) was assayed. The following primers were used: For 5'-TTTCTTGCCAGTCTGGAC-3', Rev 5'-CACGAACCACGGCACTGATT -3'. The PCR cycling conditions were: $10 \mathrm{~min}$ at $95^{\circ} \mathrm{C}, 40$ cycles of 10 $\mathrm{s}$ at $95^{\circ} \mathrm{C}$, and $1 \mathrm{~min}$ at $60^{\circ} \mathrm{C}$. Applied Biosystems 7900 system software was used for analysis. $\mathrm{Ct}$ values were determined for the internal control $(T b p)$ and Runx 2 at the same threshold in the exponential phase of PCR curves. Relative quantification (comparative $\mathrm{Ct}(\Delta \Delta \mathrm{Ct})$ method) was used to compare the expression levels. Dissociation curve analysis was performed after every run to check the specificity of the reaction. For each analysis, 3-5 reactions (each in triplicate) were run, and a standard deviation was calculated.

\section{Study on expression pattern of inflammatory cytokines and receptors by RT2 profiler assay}

Cell pellets were re-suspended in TRIzol Reagent (Sigma-Aldrich). Using RNeasy Mini kit (Qiagen) total RNA was isolated from cells, following the manufacturer's instructions. $2 \mu \mathrm{g}$ of total RNA was used to prepare cDNA using $\mathrm{RT}^{2}$ first strand kit (Qiagen). The $\mathrm{RT}^{2}$ Profiler PCR Array for rat inflammatory cytokines and receptors (SA Bioscience, Frederick, MD, USA) was used to determine the expression pattern of 84 genes involved in immune response. The q-PCR was performed, using $2 \mu \mathrm{g}$ of cDNA and Real Time PCR System 7300 (Applied Biosystems). The obtained CT values were uploaded on the manufacturer website for online analysis of gene expression.

\section{Splenocyte preparation}

Permission to perform specified procedures on rats and SCID mice was obtained by the Solna court decision (number 290/11 from August, 31th, 2011 and 192/14 from October 9th, 2014, respectively).

Spleens were harvested from the SD rats (Scanbur $\mathrm{AB})$. They were placed in a petri dish and cut into 2-3 pieces in $4 \mathrm{ml}$ of IMDM medium. Collagenase (400 U/ $\mathrm{ml}$ ) was added, and the mixture was incubated at $37^{\circ} \mathrm{C}$ for $25 \mathrm{~min}$. EDTA was immediately added at a final concentration of $2 \mu \mathrm{M}$, and the mixture was incubated for 5 min more. The spleens were mashed, and the suspension was collected in a $15 \mathrm{ml}$ Falcon tube to wash the cells. Subsequently, $10 \mathrm{ml}$ of a lysis buffer (BD Biosciences) was incubated with the pellet at room temperature for 2 min. After washing, the pellet was resuspended in $5 \mathrm{ml}$ of RPMI and passed through a cell strainer to obtain a single cell suspension of splenocytes.

Similarly, splenocytes were isolated from 6 spleens of SCID mice, 2 spleens for each experiment. After 4 days of activation by IL-2, NK cells were separated from spleenocytes using NK Cell Isolation Kit II, mouse (Miltenyi Biotec Inc, USA).

\section{Cytotoxicity and NKp46 receptor blocking assay}

Splenocytes were activated with $1000 \mathrm{U} / \mathrm{ml}$ of IL-2 for $42 \mathrm{~h}$ (4 days in the case of mouse cells) and 
were used as effectors in a standard ${ }^{52} \mathrm{Cr}$ release assay. As control target cells, the mouse lymphoma cell line YAC-1 was used. To block the NKp46 receptor, effectors were treated with anti-rat mouse monoclonal antibody WEN23 (whole IgG) that was a generous gift from Eric Dissen (Department of Anatomy, Institute of Basic Medical Sciences, University of Oslo, Norway, described in ref. [27]). Mouse IgG2a type KIR2DL2/L3 antibody (BioLegend) was used as an isotype control. In these assays, antibodies were used at the concentration $10 \mu \mathrm{g} /$ $\mathrm{ml}$.

\section{Study on 18IM cells tumorigenicity in SCID mice and immunohistochemistry}

The $1-2 \cdot 10^{6}$ of $18 \mathrm{IM}$ cells were inoculated into SCID mice subcutaneously. After 7 days mice were sacrificed and biopsies were fixed in a neutral buffered $4 \%$ formaldehyde solution. After fixation, dehydration, and embedding in paraffin, serial sections were cut at a normal thickness of $5 \mu \mathrm{m}$. Paraffin was dissolved in xylol and the tissue was rehydrated by stepwise washing with EtOH in PBS $(99 \%, 90 \%, 70 \%$, and 30\% EtOH). Tissues were then treated in a $2 \%$ solution of $\mathrm{H}_{2} \mathrm{O}_{2}$ in methanol at room temperature for $30 \mathrm{~min}$ to reduce background staining. Epitopes were exposed by hot citrate buffer (water bath, $92^{\circ} \mathrm{C}$ for $15 \mathrm{~min}$ ). Rabbit polyclonal anti-S18-2 (Proteintech Group Inc) and rat anti-mouse NKp46 (CD335) (clone 29A1.4, BioLegend) antibodies were diluted (1:200 and 1:100) in blocking buffer $(2 \%$ bovine serum albumin, $0.2 \%$ Tween-20, 10\% glycerol, and $0.05 \%$ NaN3 in PBS). The NKp46 and S18-2 signals were visualized with the help of the EnVision ${ }^{\mathrm{TM}}$ Detection systems (Dako) by Peroxidase/DAB and Permanent Red, respectively. Nuclei were stained with the Mayer's hematoxylin (Dako).

\section{Author contributions}

MM planned and performed experiments, analyzed data and took part in writing the manuscript; PNP and SD planned and performed experiments, and analyzed data; ED prepared specialized reagents and discussed the data; EK planned all the studies, analyzed and discussed data and wrote the manuscript.

\section{CONFLICTS OF INTERESTS}

Authors declare no competing interests.

\section{GRANT SUPPORT}

This work was supported by the Swedish Cancer Society, by matching grants from the Concern Foundation
(Los Angeles) and the Cancer Research Institute (New York), and by Karolinska Institutet Research Foundation.

\section{Editorial note}

This paper has been accepted based in part on peerreview conducted by another journal and the authors' response and revisions as well as expedited peer-review in Oncotarget.

\section{REFERENCES}

1. Greber BJ, Boehringer D, Leibundgut M, Bieri P, Leitner A, Schmitz N, Aebersold R, Ban N. The complete structure of the large subunit of the mammalian mitochondrial ribosome. Nature. 2014; 515: 283-286.

2. Kashuba E, Pavan Yenamandra S, Deoram Darekar S, Yurchenko M, Kashuba V, Klein G, Szekely L. MRPS18-2 protein immortalizes primary rat embryonic fibroblasts and endows them with stem cell-like properties. Proc Natl Acad Sci U S A. 2009; 106: 19866-19871.

3. Yenamandra SP, Darekar SD, Kashuba V, Matskova L, Klein G, Kashuba E. Stem cell gene expression in MRPS182-immortalized rat embryonic fibroblasts. Cell Death Dis. 2012; 3: e357.

4. Darekar SD, Mushtaq M, Gurrapu S, Kovalevska L, Drummond C, Petruchek M, Tirinato L, Di Fabrizio E, Carbone E, Kashuba E. Mitochondrial ribosomal protein S18-2 evokes chromosomal instability and transforms primary rat skin fibroblasts. Oncotarget. 2015; 6: 2101621028. doi: 10.18632/oncotarget.4123.

5. Puchtler H, Meloan SN, Terry MS. On the history and mechanism of alizarin and alizarin red S stains for calcium. J Histochem Cytochem. 1969; 17: 110-124.

6. Dalle Carbonare L, Innamorati G, Valenti MT. Transcription factor Runx2 and its application to bone tissue engineering. Stem Cell Rev. 2012; 8: 891-897.

7. Drukker M, Katz G, Urbach A, Schuldiner M, Markel G, Itskovitz-Eldor J, Reubinoff B, Mandelboim O, Benvenisty N. Characterization of the expression of MHC proteins in human embryonic stem cells. Proc Natl Acad Sci U S A. 2002; 99: 9864-9869.

8. Raulet DH, Gasser S, Gowen BG, Deng W, Jung H. Regulation of ligands for the NKG2D activating receptor. Annu Rev Immunol. 2013; 31: 413-441.

9. Ljunggren $\mathrm{HG}$, Karre K. Host resistance directed selectively against H-2-deficient lymphoma variants. Analysis of the mechanism. J Exp Med. 1985; 162: 1745-1759.

10. Karre K, Ljunggren HG, Piontek G, Kiessling R. Selective rejection of $\mathrm{H}$-2-deficient lymphoma variants suggests alternative immune defence strategy. Nature. 1986; 319 : 675-678.

11. Karre K, Ljunggren HG, Piontek G, Kiessling R. Selective rejection of $\mathrm{H}$-2-deficient lymphoma variants suggests 
alternative immune defence strategy. 1986. J Immunol. 2005; 174: 6566-6569.

12. Tallerico R, Todaro M, Di Franco S, Maccalli C, Garofalo C, Sottile R, Palmieri C, Tirinato L, Pangigadde PN, La Rocca R, Mandelboim O, Stassi G, Di Fabrizio E, et al. Human NK cells selective targeting of colon cancerinitiating cells: a role for natural cytotoxicity receptors and MHC class I molecules. J Immunol. 2013; 190: 2381-2390.

13. Jewett A, Tseng HC, Arasteh A, Saadat S, Christensen RE, Cacalano NA. Natural killer cells preferentially target cancer stem cells; role of monocytes in protection against NK cell mediated lysis of cancer stem cells. Curr Drug Deliv. 2012; 9: 5-16.

14. Dressel R, Schindehutte J, Kuhlmann T, Elsner L, Novota P, Baier PC, Schillert A, Bickeboller H, Herrmann T, Trenkwalder C, Paulus W, Mansouri A. The tumorigenicity of mouse embryonic stem cells and in vitro differentiated neuronal cells is controlled by the recipients' immune response. PLoS One. 2008; 3: e2622.

15. Kruse V, Hamann C, Monecke S, Cyganek L, Elsner L, Hubscher D, Walter L, Streckfuss-Bomeke K, Guan K, Dressel R. Human Induced Pluripotent Stem Cells Are Targets for Allogeneic and Autologous Natural Killer (NK) Cells and Killing Is Partly Mediated by the Activating NK Receptor DNAM-1. PLoS One. 2015; 10: e0125544.

16. Bruserud O, Kittang AO. The chemokine system in experimental and clinical hematology. Curr Top Microbiol Immunol. 2010; 341: 3-12.

17. Bruserud O. The Chemokine System in Experimental and Clinical Hematology: Springer Science \& Business Media; 2010. 208 p.

18. Maghazachi AA, Al-Aoukaty A, Schall TJ. CC chemokines induce the generation of killer cells from CD56+ cells. Eur J Immunol. 1996; 26: 315-319.

19. Maghazachi AA, Al-Aoukaty A. Chemokines activate natural killer cells through heterotrimeric G-proteins: implications for the treatment of AIDS and cancer. FASEB J. 1998; 12: 913-924.
20. Hanna J, Mussaffi H, Steuer G, Hanna S, Deeb M, Blau H, Arnon TI, Weizman N, Mandelboim O. Functional aberrant expression of CCR2 receptor on chronically activated NK cells in patients with TAP-2 deficiency. Blood. 2005; 106: 3465-3473.

21. Glas R, Franksson L, Une C, Eloranta ML, Ohlen C, Orn A, Karre K. Recruitment and activation of natural killer (NK) cells in vivo determined by the target cell phenotype. An adaptive component of NK cell-mediated responses. J Exp Med. 2000; 191: 129-138.

22. Robinson LA, Nataraj C, Thomas DW, Cosby JM, Griffiths R, Bautch VL, Patel DD, Coffman TM. The chemokine CX3CL1 regulates NK cell activity in vivo. Cell Immunol. 2003; 225: 122-130.

23. Pittenger MF, Mackay AM, Beck SC, Jaiswal RK, Douglas R, Mosca JD, Moorman MA, Simonetti DW, Craig S, Marshak DR. Multilineage potential of adult human mesenchymal stem cells. Science. 1999; 284: 143-147.

24. Gregory CA, Gunn WG, Peister A, Prockop DJ. An Alizarin red-based assay of mineralization by adherent cells in culture: comparison with cetylpyridinium chloride extraction. Anal Biochem. 2004; 329: 77-84.

25. Hwang KC, Kim JY, Chang W, Kim DS, Lim S, Kang SM, Song BW, Ha HY, Huh YJ, Choi IG, Hwang DY, Song H, Jang Y, et al. Chemicals that modulate stem cell differentiation. Proc Natl Acad Sci U S A. 2008; 105: 74677471.

26. Kramer J, Hegert C, Guan K, Wobus AM, Muller PK, Rohwedel J. Embryonic stem cell-derived chondrogenic differentiation in vitro: activation by BMP-2 and BMP-4. Mech Dev. 2000; 92: 193-205.

27. Westgaard IH, Berg SF, Vaage JT, Wang LL, Yokoyama WM, Dissen E, Fossum S. Rat NKp46 activates natural killer cell cytotoxicity and is associated with FcepsilonRIgamma and CD3zeta. J Leukoc Biol. 200476: 1200-1206. 